\title{
Parental misperception of their child's weight status and how weight underestimation is associated with childhood obesity
}

\author{
Daniela Rodrigues ${ }^{1,2}$ (1) \\ Aristides M. Machado-Rodrigues ${ }^{1,3}$ \\ Cristina Padez ${ }^{1,2}$ (ㅇ)
}

${ }^{1}$ CIAS-Research Centre for Anthropology and Health, University of Coimbra, Calçada Martim de Freitas, Coimbra, Portugal

${ }^{2}$ Department of Life Sciences, Faculty of Sciences and Technology, University of Coimbra, Calçada Martim de Freitas, Coimbra, Portugal

${ }^{3}$ High School of Education of Viseu, IPV, Rua Dr. Maximiano Aragão, Viseu, Portugal

\section{Correspondence}

Daniela Rodrigues, CIAS-Research Centre for Anthropology and Health, University of Coimbra, Calçada Martim de Freitas, Ed. São Bento, 3000-456

Coimbra, Portugal.

Email: rodrigues1323@gmail.com

\section{Funding information}

Foundation for Science and Technology, Grant/Award Number: SFRH/

$\mathrm{BD} / 90737 / 2012$

\begin{abstract}
Objectives: Obesity is a major public health concern worldwide. This study aims to investigate the accuracy of parental perception of child's weight and related factors as well as how underestimation is associated with the prevalence of childhood obesity.

Methods: Data from 793 parents and respective children (6-10 years) were collected during the spring of 2013 and 2014. Height and weight were measured and used to calculate body mass index and obesity was classified using the International Obesity Task Force. Parents' perception of child's weight and socio-demographic characteristics were accessed by a questionnaire. Statistical tests were used, controlling for biological and social covariates.

Results: About 33\% of parents misperceived their child's weight, of which $93 \%$ underestimated it. Different factors were found associated with greater parental underestimation according to children's weight status (ie, younger age) and sex (ie, higher mother's BMI, lower parental education, and household income for girls; living in an urban place for boys). Underestimation among girls, compared to boys, was more associated with socio-economic features of the family. For both sexes, children with excess weight were more likely to be underestimated by their parents. Parents who underestimated their child's weight were 10 to 20 times more likely to have an obese child.

Conclusions: Findings suggest a social desirability bias in parental reports of child weight status. While some parents recognize their child's weight, others may feel embarrassed to discuss the fact that their child is overweight/obese and may feel reluctant in seeking the advice of a health care professional.
\end{abstract}

\section{1 | INTRODUCTION}

Childhood obesity poses a major health concern globally, with the latest data reporting between 50 and 74 million children with obesity (NCD-RisC, 2017). In Portugal, approximately one-third of 6 to 11-years-old children have excess weight or obesity, having one of the highest rates in Europe (Rodrigues, Padez, \& Machado-Rodrigues, 2018;
Wijnhoven et al., 2014). Since obesity is associated with a number of physical health problems both immediate and in the long term (Freedman, Mei, Srinivasan, Berenson, \& Dietz, 2007; Reilly \& Kelly, 2011), and psychological health (Rankin et al., 2016), many international health agencies/societies are improving their effort on familial health education, particularly on prevention and/or lifestyle changes of adults and their kids (Gentile et al., 2018). 
Children typically look to their parents as role models regarding both their food and physical activity behaviors. In order to motivate parents and children to effectively change those behaviors, parents must first accurately perceive their child as overweight. As a first step in preventing pediatric obesity, He and Evans (2007) called for effective strategies for helping health care providers to increase parents' awareness of their children's weight status and weight problems that may be associated with overweight and obesity. However, previous studies reported that approximately $50 \%$ of parents gave an inaccurate estimating of their child's overweight status (Almoosawi et al., 2016; Lundahl, Kidwell, \& Nelson, 2014; Remmers et al., 2014; Tompkins, Seablom, \& Brock, 2015).

The literature have documented different factors that can influence the accuracy of parental perception of their child's body weight status, such as the child's sex and age, as well as the weight status and education level of the parents (De Hoog, Stronks, van Eijsden, Gemke, \& Vrijkotte, 2012; Doolen, Alpert, \& Miller, 2009; Queally, Doherty, Matvienko-Sikar, et al., 2018; Remmers et al., 2014; Rietmeijer-Mentink, Paulis, van Middelkoop, Bindels, \& van der Wouden, 2013; Rivera-Soto and Rodríguez-Figueroa, 2012; Twarog, Politis, Woods, Daniel, \& Sonneville, 2016; Yao \& Hillemeier, 2012). Studies have also identified other factors that may help to explain the parental misperception of their child's weight, such as, recent trends of increasing body size in the general population may have changed the idea of what constitutes a normal weight (Robinson, 2017), cultural beliefs about the body size (Adams, Quinn, \& Prince, 2005), not knowing the true definition of overweight, or unwillingness to accept that their child is overweight for fear of being judged as a bad parent (Turner, Salisbury, \& Shield, 2012). In all human societies, body norms matter and while the population has become heavier, cultural idealization of a "perfect body" has not changed.

Understanding the relationships of obesity and body size perceptions can lead to self-reflection about the interaction between socio-cultural messages and negative fat stigma and, therefore, it might be used to improve obesity prevention programs. Still, most of the studies in this field have been conducted in the United States, Australia, or UK and usually just includes the mothers' perception of the child's weight (Lopes, Santos, Pereira, \& Lopes, 2012; Rietmeijer-Mentink et al., 2013). However, body concerns or ideals might adjust to the living context and changes in employment patterns and family structure make it important to observe both mother's and father's perceptions. Therefore, the purpose of the current study was 2-fold: (a) to explore the agreement between the body mass index (BMI)-based weight status and parental perception of children's weight and (b) to observe if that perception was associated with childhood obesity. Research questions are: (a) how accurate parents perceive their children's weight status and the direction of the discordant weight (overestimation vs underestimation), (b) how the misperception varies across different child and socio-economic characteristics, and (c) how parental underestimation of their child's weight impacts children health-related physical outcomes (ie, overweight and obesity).

\section{2 | METHODS}

\section{1 | Study design and sampling}

This study derived from a larger project designed to determine social, cultural, and physical factors associated with child participation in extracurricular sport and obesity rates in Portuguese elementary school children. The study adopted a cross-sectional design and was conducted in central Portugal during 2013 and 2014 from April to mid-June. Ethical and protocol approval was given by the Portuguese Commission for Data Protection which requires anonymity and no transmissibility of data, and corroborated by the Direcção Geral de Inovação e Desenvolvimento Curricular (Portuguese General Directorate of the Ministry of Education). The study protocol was in accordance with the International Ethical Guidelines for Biomedical Research Involving Human Subjects and followed the ethical standards of the Helsinki Declaration (1975/2008). Parents were fully informed about all of the study procedures. Written informed consent was obtained and confidentiality of all the collected and archived data was ensured. Children's own consent was obtained prior to the anthropometric measurements.

Questionnaires were delivered to all the parents in six primary schools from the central of mainland Portugal $(n=1369)$. Parents were given 1 week to return the questionnaires completely filled if they wanted to participate in the study. The questionnaire was adapted from another one previously used in the same population to study active behaviors in children (Machado-Rodrigues et al., 2014). Parents who reported some health condition that could hinder their children's participation in sport activities were not included further in the study. A total of 834 parents delivered completely fill questionnaires and consent forms; 793 children (aged 6-10-years-old; 424 girls) were included in the measurement stage (eg, some children were not at school during the data collection, some were sick, or did not wanted to participate; response rate $=57.9 \%$ ).

\section{$2.2 \mid$ Measures}

Anthropometric measurement was collected by a trained investigator and according to standardized procedures in 
light indoor clothing and without shoes. Height and weight were recorded to the nearest $0.1 \mathrm{~cm}$ and $100 \mathrm{~g}$, respectively. BMI was subsequently calculated and weight status was classified according to the sex- and age-specific World Obesity Federation (WOF, former International Obesity Task Force, IOTF) cutoff points for BMI (Cole, Bellizzi, Flegal, \& Dietz, 2000; Cole \& Lobstein, 2012).

Parental classification of children's weight was assessed with the question "How would you describe your child's weight at the moment? Possible answers were one of the following four choices: "My child's weight is a bit too little," "My child's weight is OK," "My child's weight is a bit too much," and "My child's weight is way too much." For the logistic analysis, underestimation of child's weight was considered instead of an inaccurate perception. Since child's nutritional status and parental classification were registered from 1 to 4 (from underweight to obesity, respectively), the scores were subtracted and positive scores represented underestimation of child's weight. Similar procedure was used in other epidemiological studies (Manios et al., 2015).

The questionnaire had different sections regarding mothers and fathers' characteristics. One of the questions included information about parents' weight and height. Parental BMI was calculated and the cutoff points of the World Health Organization (WHO) were used to classified mothers and fathers as having underweight, normal weight, overweight, or obesity (WHO, 2000). Parental BMI was later divided into two groups, namely: (a) underweight and normal weight (if BMI was equal or below $24.99 \mathrm{~kg} / \mathrm{m}^{2}$ ) and (b) overweight and obesity (if BMI was greater than $25.00 \mathrm{~kg} / \mathrm{m}^{2}$ ).

Age was divided into two groups: 6 to 7 years and 8 to 10 -years-old. This division was chosen for practical and physiological reasons since by the age of 6 years the adiposity rebound occurs, following the nadir of the BMI curve. This age range is also likely to be a favorable period for prevention strategies. Parents reported if their child practiced any extracurricular sport (extra academic activity), children's way of transportation to and from school (active or passive), and their children's television (TV) viewing habits ( $<2$ hours/ day vs $\geq 2$ hours/day). Mean monthly household income was defined as low ( $<€ 1000$ per month), medium (€1000-€1500/month) or high ( $>€ 1500 /$ month). Education level of the parents was used to complement the socio-economic status (SES) measure (one or none parent have a university vs both parents have a university degree), since Portugal does not have an official classification for economic status. Place of residence was classified according to the Portuguese Statistical System criteria (Monteiro, 2000).

\section{3 | Statistical analysis}

Descriptive analysis and $\chi^{2}$ tests were conducted to describe the characteristics of the participants, according to children's sex. Weighted kappa was calculated to measure the agreement between parental weight perception and child's BMI-based weight status. Kappa values are commonly classified in six categories: poor agreement $(K<0.00)$, slight agreement $(K=0.00-0.20)$, fair agreement $(K=0.21-0.40)$, moderate agreement $(K=0.41-0.60)$, substantial agreement $(K=0.61-0.80)$, and almost perfect agreement $(K=0.81-1.0)$. The weight perception rate was described. Two logistic regression models were constructed, one according to the children's weight status and the other according to children's sex, in order to analyze the association between the parental underestimation and child/socio-economic factors. A final logistic regression model was created to observe the variables associated with childhood overweight, including obesity. The analyses were performed using the Statistical Package for the Social Sciences (SPSS v.23; SPSS an IBM Company, Chicago, Illinois).

\section{3 | RESULTS}

Child and socio-demographic characteristics of the sample are presented in Table 1 . The mean age of the children was $8.05 \pm 1.21$ years. Significantly more girls than boys were overweight (17.4\% and $14.3 \%$, respectively) or obese (7.6\% and $4.4 \%$, respectively). A total of 619 mothers and 174 fathers responded to the questionnaire. No statistical differences were found for parental education or income according to children's sex.

A total of 261 (32.9\%) parents incorrectly classified their child's weight, including $30.6 \%$ of parents who underestimated their child's weight status from normal to underweight, from overweight to normal weight, and from obesity to overweight. The poor agreement of parents' perception and child's weight status was confirmed by the kappa coefficient $(K=0.231, P<.001)$, which was slightly greater among girls $(K=0.239)$ than among boys $(K=0.224)$ (Figure 1$)$. No significant differences were found on parental misperception according to children's sex (girls $=33.8 \%$, boys $=32.0 \%$ ) but a $P$-value of $<.001$ was found according to children's real weight status, in both girls and boys.

Tables 2 and 3 show the variables associated with the odds of parental underestimation of children's weight, according to their children's sex and BMI status. Parents of boys were more likely to underestimate their child's weight if they lived in urban communities $(\mathrm{OR}=1.70$, $P=.02)$ and if the child had excess weight $(\mathrm{OR}=10.85$, 


\begin{tabular}{|c|c|c|c|c|c|c|}
\hline \multicolumn{3}{|c|}{ Sample characteristics } & \multirow{2}{*}{$\begin{array}{l}\mathbf{N} \\
424\end{array}$} & \multirow{2}{*}{$\begin{array}{l}\% \\
50.8\end{array}$} & \multirow{2}{*}{$\begin{array}{l}\text { Girls } \\
\mathrm{n} / \mathrm{a}\end{array}$} & \multirow{2}{*}{$\begin{array}{l}\text { Boys } \\
\text { n/a }\end{array}$} \\
\hline \multirow[t]{14}{*}{ Child } & \multirow[t]{2}{*}{ Sex } & Girls & & & & \\
\hline & & Boys & 410 & 49.2 & $\mathrm{n} / \mathrm{a}$ & $\mathrm{n} / \mathrm{a}$ \\
\hline & \multirow[t]{2}{*}{ Age } & 6-7 years & 325 & 39.0 & 36.8 & 41.2 \\
\hline & & $8-10$ years & 509 & 61.0 & 63.2 & 58.8 \\
\hline & \multirow[t]{4}{*}{$\mathrm{BMI}^{\mathrm{a}}$} & Underweight & 26 & 3.3 & 4.2 & 2.3 \\
\hline & & Normal weight & 593 & 74.8 & 70.8 & 79.0 \\
\hline & & Overweight & 126 & 15.9 & 17.4 & 14.3 \\
\hline & & Obesity & 48 & 6.1 & 7.6 & 4.4 \\
\hline & \multirow[t]{2}{*}{ Commute } & Active & 166 & 19.9 & 20.3 & 19.5 \\
\hline & & Passive & 668 & 80.1 & 79.7 & 80.5 \\
\hline & \multirow[t]{2}{*}{ Practices sport } & Yes & 565 & 67.7 & 67.2 & 68.5 \\
\hline & & No & 269 & 32.3 & 32.8 & 31.5 \\
\hline & \multirow[t]{2}{*}{ TV viewing } & $<2$ hours/day & 230 & 28.2 & 28.1 & 28.3 \\
\hline & & $\geq 2$ hours/day & 586 & 71.8 & 71.9 & 71.8 \\
\hline \multirow[t]{13}{*}{ Parent } & \multirow[t]{2}{*}{ Mother's BMI } & Under/Normal weight & 540 & 42.4 & 69.2 & 70.9 \\
\hline & & Overweight/Obesity & 231 & 57.6 & 30.8 & 29.1 \\
\hline & \multirow[t]{2}{*}{ Father's BMI } & Under/Normal weight & 311 & 42.4 & 45.2 & 39.6 \\
\hline & & Overweight/Obesity & 422 & 57.6 & 54.8 & 60.4 \\
\hline & \multirow[t]{2}{*}{ Mother education } & $<$ University degree & 370 & 45.5 & 43.3 & 58.8 \\
\hline & & University degree & 444 & 54.5 & 56.7 & 41.2 \\
\hline & \multirow[t]{2}{*}{ Father education } & $<$ University degree & 465 & 58.3 & 57.8 & 47.6 \\
\hline & & University degree & 333 & 41.7 & 42.2 & 52.4 \\
\hline & \multirow[t]{3}{*}{ Household income } & $<€ 1000 /$ month & 234 & 29.7 & 29.9 & 29.5 \\
\hline & & $€ 1000-€ 1500 /$ month & 206 & 26.1 & 25.4 & 26.9 \\
\hline & & $>€ 1500 /$ month & 348 & 44.2 & 44.8 & 43.5 \\
\hline & \multirow[t]{2}{*}{ Residence } & Urban setting & 443 & 53.1 & 54.5 & 51.7 \\
\hline & & Non-urban setting & 391 & 46.9 & 45.5 & 48.3 \\
\hline
\end{tabular}

Note: Denotes $P<.001 ;$ denotes $P<.01 ;{ }^{\text {a }}$ denotes $P<.05$ using $\chi^{2}$ tests. n/a: not applicable. Children's BMI was classified using WOF cutoffs specific for sex and age (former IOTF, Cole et al., 2000; Cole \& Lobstein, 2012). Parents' BMI was classified using WHO (2000) criteria (below 18.5: underweight, 18.5-24.9: normal weight, 25.0-29.9: overweight, above 30.0: obesity).
T A B L E 1 Demographic and behavioral characteristics of the sample

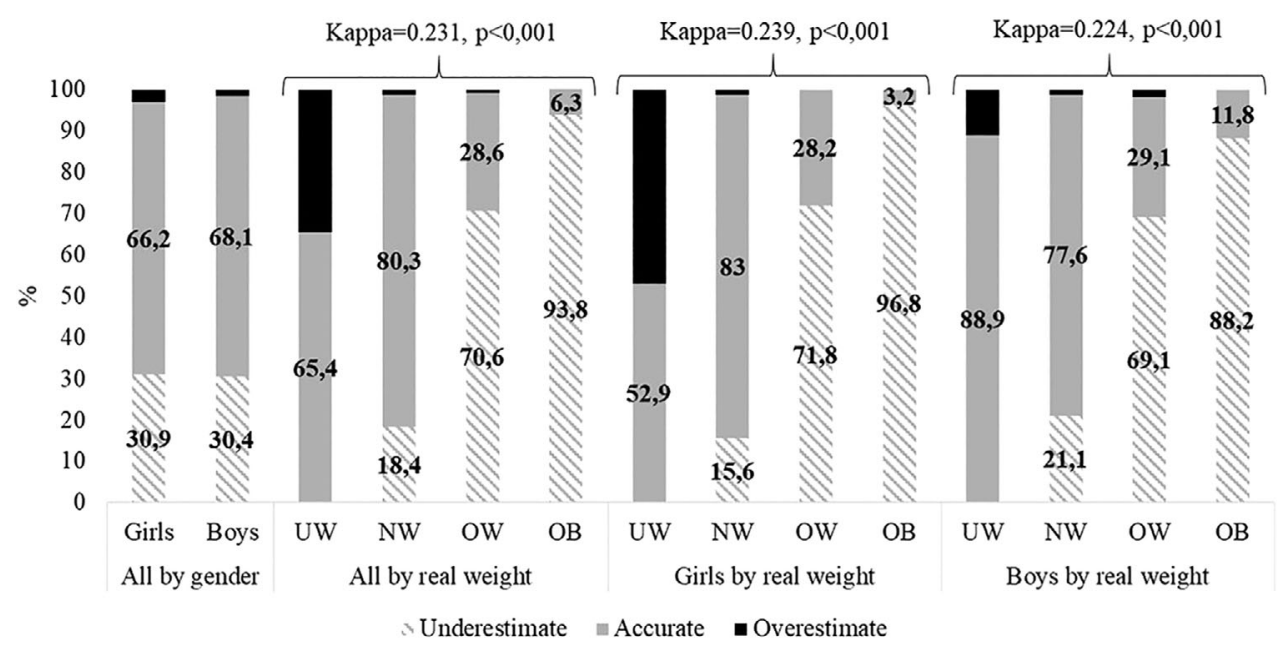

FIGURE 1 Comparison of parental body weight perception by children's sex and real weight among Portuguese children. Children's weight was defined by the WOF cutoffs (Cole et al., 2000; Cole \& Lobstein, 2012). OB, obesity; OW, overweight; NW, normal weight; UW, underweight. All by sex: $P=.41$, all by real weight: $P<.001$, girls by real weight: $P<.001$, boys by real weight: $P<.001$ 
T A B L E 2 Logistic regression of the association between both parents' underestimation of children's weight status, according to children's sex

\begin{tabular}{|c|c|c|c|}
\hline Independent variables & & \multicolumn{2}{|c|}{ OR (CI 95\%) for parental underestimation of children's weight } \\
\hline \multirow[t]{2}{*}{ Children's age } & $6-7$ years & $0.84(0.54-1.30)$ & $1.25(0.80-1.93)$ \\
\hline & $8-10$ years & Ref. & \\
\hline Children's BMI & Over/Obesity & $22.37(12.59-39.75)^{\mathrm{a}}$ & $10.85(6.01-19.61)^{\mathrm{a}}$ \\
\hline Respondent & Father & Ref. & \\
\hline \multirow[t]{2}{*}{ Mother's BMI } & Over/obesity & $1.85(1.16-2.94)^{\mathrm{b}}$ & $1.34(0.82-2.20)$ \\
\hline & Under/normal & Ref. & \\
\hline Father's BMI & Over/obesity & $0.76(0.48-1.20)$ & $1.15(0.72-1.86)$ \\
\hline Parental education & University & Ref. & \\
\hline \multirow[t]{3}{*}{ Household income } & Low & $1.92(1.15-3.19)^{\mathrm{c}}$ & $0.74(0.42-1.29)$ \\
\hline & Medium & $1.67(0.97-2.85)$ & $1.44(0.84-2.45)$ \\
\hline & High & Ref. & \\
\hline \multirow[t]{2}{*}{ Residence } & Urban & $0.96(0.63-1.46)$ & $1.70(1.09-2.65)^{\mathrm{c}}$ \\
\hline & Non-urban & Ref. & \\
\hline
\end{tabular}

Note: Parental education was classified as: (a) no parent, or just one, have a university degree, (b) both parents have a university degree. BMI, body mass index. Parental weight status was classified using the WHO (2000) criteria.

a Denotes $P<.001$.

${ }^{\mathrm{b}}$ Denotes $P<.01$.

${ }^{\mathrm{c}}$ Denotes $P<.05$.

$P<.001$; Table 2). Furthermore, parents of girls had significantly higher odds of underestimate their daughter's weight: (a) if the child was overweight/obese (vs normal weight; $\mathrm{OR}=22.37, P<.001$ ); (b) if the mother was overweight/obese (vs normal weight; $\mathrm{OR}=1.85, P=.01$ ); (c) if none or only one parent had a university degree (vs both parents holding a university degree; OR $=2.09$, $P=.00)$; and (d) if the family reported a low income (vs medium or high income; $\mathrm{OR}=1.92, P=.01$ ). Children's age was associated with parental underestimation but not in all BMI categories namely, parents of younger children had more than three times the odds to underestimate their child's weight if their children were overweight or obese, in comparison with parents of older children $(P=.01$; Table 3$)$.

As shown in Table 4, the greatest predictor for childhood obesity, in both the crude and the adjusted model, was the parental underestimation of their child's weight status, with children having more than 20 (for girls) and more than 10 times the odds (for boys), to be classified with overweight if their parents underestimate their weight. In addition, among girls aged 6 to 10 years, TV watching, parental education, mother's BMI, and household income were the significant predictors of obesity in the crude model; corresponding factors for boys were the active commuting, the mother's BMI, and the household income. However, after controlling for the aforementioned confounding factors, most of the associations lost their statistical significance.

\section{4 | DISCUSSION}

This study shows that one in three parents misperceived their child's weight (30.6\% underestimate and $2.3 \%$ overestimate) which is somewhat in line with previous studies that examined weight misperception patterns among parents of children in different populations (Lundahl et al., 2014; Rietmeijer-Mentink et al., 2013; Tompkins et al., 2015). Studies in Asian countries like China, showed weight misperception rates around 40\% (Zhang, Cai, Jing, Ma, \& Chen, 2018) and a recent study in Brazil revealed that $48.05 \%$ of parents incorrectly classified their child's weight (Warkentin, Mais, Latorre, Carnell, \& Taddei, 2018). Lower 
T A B L E 3 Logistic regression of the association between both parents' underestimation of children's weight status, according to children's weight status

\begin{tabular}{|c|c|c|c|}
\hline & & \multicolumn{2}{|c|}{ OR (CI 95\%) for parental underestimation of children's weight } \\
\hline Children's sex & Boys & Ref. & \\
\hline Children's age & $6-7$ years & $0.84(0.55-1.29)$ & $3.39(1.40-8.20)^{\mathrm{a}}$ \\
\hline Respondent & Father & Ref. & \\
\hline \multirow[t]{2}{*}{ Mother's BMI } & Over/obesity & $0.84(0.51-1.40)$ & $2.04(0.95-4.39)$ \\
\hline & Under/normal & Ref. & \\
\hline Father's BMI & Over/obesity & $0.81(0.52-1.27)$ & $0.85(0.39-1.85)$ \\
\hline Parental education & University & Ref. & \\
\hline \multirow[t]{3}{*}{ Household income } & Low & $0.76(0.45-1.27)$ & $1.78(0.76-4.15)$ \\
\hline & Medium & $1.01(0.60-1.69)$ & $2.32(0.93-5.76)$ \\
\hline & High & Ref. & \\
\hline \multirow[t]{2}{*}{ Residence } & Urban & $1.39(0.91-2.11)$ & $0.76(0.37-1.58)$ \\
\hline & Non-urban & Ref. & \\
\hline
\end{tabular}

Note: Parental education was classified as: (a) no parent, or just one, have a university degree, (b) both parents have a university degree. BMI, body mass index. Parental weight status was classified using the WHO (2000) criteria. denotes $P<.001$; ${ }^{\text {a }}$ denotes $P<.01$; denotes $P<.05$.

rates of misperception (26.3\%) were found in a representative sample of U.S. parents of children and adolescents (Chen et al., 2014), and in a sample of 0 to 14-years-old children from Austria (24.3\%; Nemecek, Sebelefsky, Woditschka, \& Voitl, 2017).

Parental underestimation was particularly high among children with excess weight and obesity, which is in line with previous studies (Lundahl et al., 2014; Nemecek et al., 2017; Parkinson et al., 2017; RietmeijerMentink et al., 2013). This result is particularly important considering the high prevalence of childhood obesity worldwide, including in Portugal (Rodrigues et al., 2018; Wijnhoven et al., 2014), and the health implications of this condition (Freedman et al., 2007; Rankin et al., 2016; Reilly \& Kelly, 2011). The percentage of total agreement between the child's objectively measured weight status and parental perception of the child's weight found in the present study $(67.1 \%)$ is lower than the rate found by Lopes and colleagues (2012) in a sample of Portuguese children aged 9 to 12 -years-old (71.1\%). In addition, that research revealed that about $62 \%$ of overweight/obese children had their weight underestimate by their mother (Lopes et al., 2012) while the present study registered an higher prevalence of parental underestimation in the same weight category (77.0\%). This may suggest the existence of a trend of misperception, in which the perceived normal weight has increased with the increased prevalence of obesity. A similar trend was reported by Duncan, Hansen, Wang, Yan, and Zhang (2015), who observed a 30\% reduction in the parental correct recognition was found between 1988-1994 and 2007-2012.

Data of the present study cannot show whether the parents are poorly informed about their children's weight status or well informed but knowingly underreported it (eg, social desirability bias). Parents might evaluate their child's weight status in relation to others rather than against an absolute scale, meaning that the high prevalence of children with excess weight and obesity may have led to an acceptance of excess body fat, making that condition the norm (Robinson, 2017; Suls, Martin, \& Wheeler, 2002). This social comparison theory was supported by Binkin, Spinelli, Baglio, and Lamberti (2013) in U.S. mothers residing in a region with high obesity prevalence which had a higher likelihood of failing to correctly identify their child as overweight. Moreover, media often feature objectified fat bodies passing a distorted impression of the criteria to define overweight (Campbell, Williams, Hampton, \& Wake, 2006).

On the other hand, misreported weight status among parents that know that their child is overweight/obese 
T A B L E 4 Logistic regression predicting childhood obesity (including overweight) in a sample of children aged 6-10 years living in central Portugal (2013/2014)

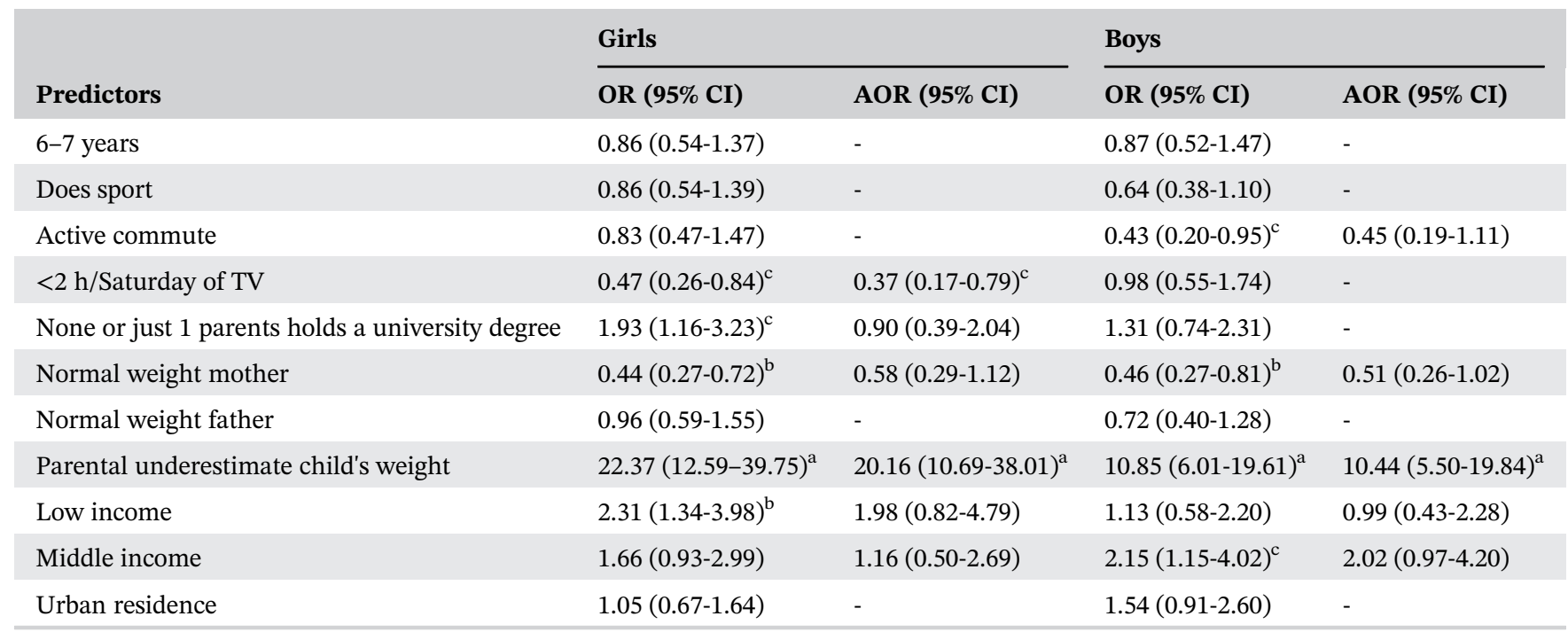

Note: AOR, adjusted odds ratio; BMI, body mass index; CI, confidence interval; OB, obesity; OR, odds ratio; OW, overweight; TV, television; parental weight status was classified using the WHO (2000) criteria. All the significant variables in the crude model (OR) were carried forward, simultaneously, to an adjusted model (AOR).

${ }^{\mathrm{a}}$ Denotes $P<.001$.

${ }^{\mathrm{b}}$ Denotes $P<.01$.

${ }^{\mathrm{c}}$ Denotes $P<.05$.

may occur because parents feel embarrassed or they not want to be blamed for their child's weight problem (Eli, Howell, Fisher, \& Nowicka, 2014; Turner et al., 2012). Parents may also avoid labeling their child as "overweight" or "obese" for fear of leading to children's stigmatization (Turner et al., 2012). In this context, stigma refers to the process by which arbitrary moral judgments (eg, lazy) become attached to body size, leading to social discrediting, rejection, and marginalization (Brewis, 2014; Brewis \& Wutich, 2019). The stigmatization of obesity, as obesity itself, is an increasing global, cultural, and biological phenomenon that often starts at a young age (Hansson, Karnehed, Tynelius, \& Rasmussen, 2009). Moreover, fat stigma is often associated with fear of fat, anti-fat attitudes, low self-esteem, negative body image, and poor quality of life among overweight and obese children (Brewis \& Wutich, 2019; Farhat, Iannotti, \& Summersett-Ringgold, 2015; Guzmán de la Garza et al., 2019; Latner, Simmonds, Rosewall, \& Stunkard, 2007; Szamreta et al., 2017).

Turner et al. (2012) found that parents who had struggled with their own weight were less receptive to consult a practitioner about their child's weight, many times remembering their own embarrassing when they were recognized as overweight or obese. This can explain why overweight parents in the present study, particularly mothers, were more likely to underestimate their child's weight status in comparison with parents with normal weight. Women, more than men, often suffer stigmatization due to their weight across multiple sectors, including employment, education, sports, and media (Fikkan \& Rothblum, 2012; Sattler, Deane, Tapsell, \& Kelly, 2018) and it may be the case that overweight mothers, knowing in first-hand the negative connotations and the stigmatization associated with the word "overweight" and "obesity," do not wish to verbally label their child as such. Social and cultural norms as well as the standards for attractiveness shown on social media may help to explain differences between sexes; however, given that those norms vary by specific cultural models and ecological contexts, differences between males and females are far to be consistent (Kelleher, Millar, Shiely, Perry, \& Harrington, 2018; Lopes et al., 2012; Queally et al., 2018; Remmers et al., 2014; Rivera-Soto \& Rodríguez-Figueroa, 2012).

Differences according to income and educational level may be related with the socioeconomic gradient in childhood obesity (Lissner et al., 2016; Manios et al., 2015; Queally et al., 2018; Rivera-Soto \& Rodríguez-Figueroa, 2012). The relationship between poverty and obesity is complex because, while poverty creates an environment conducive to fostering obesity (eg, poorer diets, less physical activity opportunities, etc.), obesity stigmatization can also facilitate poverty (eg, less job promotions, lower wages, among other factors) (McCullough \& Hardin, 2013; Puhl \& Brownell, 2001). Present findings show that 
misperception of the child's weight was higher among parents with lower education which is line with the study carried out by Jain et al. (2001) in which low-income mothers were reluctant to describe their preschool children as "overweight." Those mothers often expressed a distrust of growth charts and perceived them as having little relevance to their child. In the present study, worse educated parents were more likely to misperceive their daughter's weight, but not their sons. Similar results according to child's sex were reported in a sample of Irish young children (Queally et al., 2018) possibly indicating that parents explain body size differences between girls and boys as normal gender differences and that, in this context, they also may be influenced by the social desirability for a lower body weight in girls and for a larger body size in boys. Also, lifestyle and social pressure affecting body image might differ by level of development, which is usually higher in urban contexts, leading to greater rates of underestimation as seen in the present study. However, the reason why factors varied according to children's sex is unclear and should be further investigated in future studies.

In line with this lack of concordance between objective and perceptual measures of children's weight status, very few parents incorrectly classified their child with underweight as normal weight and their child of normal weight as overweight. Nevertheless, previous studies have observed that when a parent identifies a child as being overweight, that child may have lower self-esteem and be at increased risk of future weight gain (Robinson \& Sutin, 2017).

The second purpose of the present study investigated the factors associated with childhood obesity in order to understand the etiology of obesity and provide clues to Health Portuguese Institutions for a possible and more focused intervention among children. The results showed that the strongest predictor of childhood overweight, including obesity was parental underestimation of their child's weight. Boys had 10 times more odds of having excess weight if parents underestimate their weight and those odds doubled among girls. Similar results were found in a sample of North American children in which parents who misperceived their child's weight were almost 12 times more likely of having an obese child (McKee, Long, Southward, Walker, \& McCown, 2016). Parents who underestimate their child's weight may be less concerned about the child's nutritional status (Tschamler, Conn, Cook, \& Halterman, 2010) and if parents do not acknowledge that their child's weight can damage his or her health, interventions may be less likely to occur and necessary steps to improve their child's diet and physical activity may not be adopted (Lundahl et al., 2014).

This study has several strengths including the relatively large sample and high response rate, and the objectively measure of child's height and weight. Obesity as a phenomenon simply cannot be understood without placing it in its fuller societal context, so the use of different child and socioeconomic factors to observe parental underestimation was necessary. The observed differences according to sex may suggest culturally variable feedback and pressure on feminine over masculine bodies, which require further research. This investigation adds to a very small body of studies in the Portuguese population about parental perception of their child's weight status. Present study is not without limitations such as, parental BMI being calculated by self-reported height and weight, and not directly measured, the small $\mathrm{N}$ of fathers compared to mothers, and directionality of associations concerning parental misperception could not be determined due to the cross-sectional design. Another limitation is the use of a question to measure parental perceived weight status instead of silhouette rating scales.

Also, taking into account the present findings, it is important to bear in mind some caveats to the analysis. Judging parental perceptions as misperceptions represents the contemporary BMI classification as a culturally shared and true standard for judging "normal," but the clinically definition of normal may differ from the idealistically or culturally one (McCullough \& Hardin, 2013). Also, nothing was known about the child's BMI history and so, if the child used to be much heavier, parents may now think that they are not overweight/obese, even if they still are, and the analysis was not controlled for the weight status of the siblings, which may be used to base parental perception.

\section{5 | CONCLUSION}

Findings revealed that while some parents recognize their child's weight, other may feel embarrassed to discuss the fact that their child is overweight/obese and may feel reluctant in seeking the advice of a health care professional. Parental underestimation of boys and girls was associated with different variables that may point for social and economic factors in shaping and layering body concerns or ideals. Negative fat stigma shapes health relevant behaviors, creates suffering and mitigates health disparities and thus, requires urgent changes. Given the poorer agreement between parental perception of child's weight and child's real weight status, clinicians must be conscious that children should be weighed and measured regularly and not depend of parental assessment of weight. Also, health care providers must impart knowledge to parents about the health risks of childhood obesity and present them healthier choices for themselves and their families (ie, healthier diets, less sedentary behaviors, and physical activity habits). However, there is a need to understand the cultural and 
contextual characteristics and how those impact the ideas of body size, obesity, and health in a population and its increasing diversities.

\section{ACKNOWLEDGMENTS}

This study was funded by the Foundation for Science and Technology (FCT) under grant SFRH/BD/90737/2012. We gratefully acknowledge all the schools, parents, and children that participated in this study.

\section{DISCLOSURE OF INTEREST}

No competing financial interests exist.

\section{AUTHOR CONTRIBUTIONS}

Study concept and design: All authors. Acquisition, analysis, and interpretation of data: D.R. Statistical analysis: D.R. Drafting of the manuscript: D.R. Critical revision of the manuscript: All authors.

\section{ORCID}

Daniela Rodrigues (D) https://orcid.org/0000-0002-45594303

Aristides M. Machado-Rodrigues (1) https://orcid.org/00000002-7169-8034

Cristina Padez (10 https://orcid.org/0000-0002-1967-3497

\section{REFERENCES}

Adams, A. K., Quinn, R. A., \& Prince, R. J. (2005). Low recognition of childhood overweight and disease risk among NativeAmerican caregivers. Obesity Research, 13(1), 146-152.

Almoosawi, S., Jones, A. R., Parkinson, K. N., Pearce, M. S., Collins, H., \& Adamson, A. J. (2016). Parental perception of weight status: Influence on children's diet in the Gateshead Millenium Study. PLoS One, 11(2), e0144931.

Binkin, N., Spinelli, A., Baglio, G., \& Lamberti, A. (2013). What is common becomes normal: The effect of obesity prevalence on maternal perception. Nutrition, Metabolism \& Cardiovascular Diseases, 23(5), 410-416.

Brewis, A., \& Wutich, A. (2019). Stigma: A biocultural proposal for integrating evolutionary and political-economic approaches. American Journal of Human Biology, 31(4), e23290.

Brewis, A. A. (2014). Stigma and the perpetuation of obesity. Social Science \& Medicine, 118, 152-158.

Campbell, M. W., Williams, J., Hampton, A., \& Wake, M. (2006). Maternal concern and perceptions of overweight in Australian preschool-aged children. The Medical Journal of Australia, 184, 274-277.

Chen, H. Y., Lemon, S. C., Pagoto, S. L., Barton, B. A., Lapane, K. L., \& Goldberg, R. J. (2014). Personal and parental weight misperception and self-reported attempted weight loss in US children and adolescents, National Health and Nutrition Examination Survey, 2007-2008 and 2009-2010. Preventing Chronic Disease, 31(11), E132.

Cole, T. J., Bellizzi, M., Flegal, K. M., \& Dietz, W. H. (2000). Establishing a standard definition for child overweight and obesity worldwide: International survey. BMJ, 320, 1240-1243.
Cole, T. J., \& Lobstein, T. (2012). Extended international (IOTF) body mass index cut-offs for thinness, overweight and obesity. Pediatric Obesity, 7(4), 284-294.

De Hoog, M. L. A., Stronks, K., van Eijsden, M., Gemke, R. J. B. J., \& Vrijkotte, T. G. M. (2012). Ethnic differences in maternal underestimation of offspring's weight: The ABCD study. International Journal of Obesity, 36, 53-60.

Doolen, J., Alpert, P. T., \& Miller, S. K. (2009). Parental disconnect between perceived and actual weight status of children: A metasynthesis of the current research. Journal of the American Academy of Nurse Practitioners, 21(3), 160-166.

Duncan, D. T., Hansen, A. R., Wang, W., Yan, F., \& Zhang, J. (2015). Change in misperception of child's body weight among parents of American preschool children. Childhood Obesity, 11 (4), 384-393.

Eli, K., Howell, K., Fisher, P. A., \& Nowicka, P. (2014). “A little on the heavy side": A qualitative analysis of parents' and grandparents perceptions of pre-schoolers' body weight. BMJ, 4, e006609.

Farhat, T., Iannotti, R. J., \& Summersett-Ringgold, F. (2015). Weight perceptions, and health-related quality of life among a National sample of US girls. Journal of Developmental and Behavioral Pediatrics, 36, 313-323.

Fikkan, J. L., \& Rothblum, E. D. (2012). Is fat a feminist issue? Exploring the gendered nature of weight bias. Sex Roles, 66(9), 575-592.

Freedman, D. S., Mei, Z., Srinivasan, S. R., Berenson, G. S., \& Dietz, W. H. (2007). Cardiovascular risk factors and excess adiposity among overweight children and adolescents: The Bogalusa Heart Study. The Journal of Pediatrics, 150(1), 12-17 (e2).

Gentile, N., Kaufman, T. K., Maxson, J., Klein, D. M., Merten, S., Price, M., ... Lynch, B. A. (2018). The effectiveness of a familycentered childhood obesity intervention at the YMCA: A pilot study. Journal of Community Medicine \& Health Education, 8(1), 591.

Guzmán de la Garza, F. J., Salinas-Martínez, A. M., ZendejasValdéz, J. M., Cordero-Franco, H. F., Mathiew-Quirós, A., \& Garza-Salinas, L. H. (2019). Body frame size, body image, selfesteem, and health-related quality of life in schoolchildren. American Journal of Human Biology, 31(4), e23294.

Hansson, L. M., Karnehed, N., Tynelius, P., \& Rasmussen, F. (2009). Prejudice against obesity among 10-year-old: A nationwide population-based study. Acta Paediatrica, 98(7), 1176-1182.

He, M., \& Evans, A. (2007). Are parents aware that their children are overweight or obese? Do they care? Canadian Family Physician, 53(9), 1493-1499.

Jain, A., Sherman, S. N., Chamberlin, L. A., Carter, Y., Powers, S. W., \& Whitaker, R. C. (2001). Why don't low-income mothers worry about their pre-schoolers being overweight? Pediatrics, 107(5), 1138-1146.

Kelleher, E., Millar, S., Shiely, F., Perry, I. J., \& Harrington, J. M. (2018). Parent and child misperception of child weight status: A crosssectional analysis of the Cork children's lifestyle study (CCLaS). Journal of Epidemiology and Community Health, 72(S1), A59.

Latner, J. D., Simmonds, M., Rosewall, J. K., \& Stunkard, A. J. (2007). Assessment of obesity stigmatization in children and adolescents: Modernizing a standard measure. Obesity, 15(12), 3078-3085.

Lissner, L., Wijnhoven, T. M. A., Mehlig, K., Sjöberg, A., Kunesova, M., Yngve, A., ... Breda, J. (2016). Socioeconomic inequalities in childhood overweight: Heterogeneity across five countries in the WHO European Childhood Obesity Surveillance 
Initiative (COSI-2008). International Journal of Obesity, 40, 796-802.

Lopes, L., Santos, R., Pereira, B., \& Lopes, V. (2012). Maternal perceptions of children's weight status. Child: Care, Health and Development, 39(5), 728-736.

Lundahl, A., Kidwell, K. M., \& Nelson, T. D. (2014). Parental underestimates of child weight: A meta-analysis. Pediatrics, 133, e689-e703.

Machado-Rodrigues, A. M., Santana, A., Gama, A., Mourão, I., Nogueira, H., Rosado, V., \& Padez, C. (2014). Parental perception of neighborhood environments, BMI, and active behaviors in girls aged 7-9 years. American Journal of Human Biology, 26 (5), 670-675.

Manios, Y., Moschonis, G., Karatzi, K., Androutsos, O., Chinapaw, M., Moreno, L. A., ... Brug, J. (2015). Large proportions of overweight and obese children, as well as their parents, underestimate children's weight status across Europe. The ENERGY (EuropeaN Energy balance Research to prevent excessive weight Gain among youth) project. Public Health Nutrition, 18(12), 2183-2190.

McCullough, M. B., \& Hardin, J. A. (Eds.). (2013). Reconstructing obesity: The meaning of measures and the measures of meaning. Food, nutrition, and culture (volume 2). New York: Berghahn Books.

McKee, C., Long, L., Southward, L. H., Walker, B., \& McCown, J. (2016). The role of parental misperception of child's body weight in childhood obesity. Journal of Pediatric Nursing, 31(2), 196-203.

Monteiro, J. L. (2000). Caracterização dos espaços urbanos na RLVT: o contributo da análise discriminante. Revista de Estudos Regionais-Instituto Nacional de Estatística, 2(1), 21-46.

NCD-RisC. (2017). Worldwide trends in body-mass index, underweight, overweight, and obesity from 1975 to 2016: A pooled analysis of 2416 population-based measurement studies in 128.9 million children, adolescents, and adults. Lancet, 390, 2627-2642.

Nemecek, D., Sebelefsky, C., Woditschka, A., \& Voitl, P. (2017). Overweight in children and its perception by parents: A crosssectional observation in a general pediatric outpatient clinic. BMC Pediatrics, 17, 212-222.

Parkinson, K. N., Reilly, J. J., Basterfield, L., Reilly, J. K., Janssen, X., Jones, A. R., ... Adamson, A. J. (2017). Mothers' perceptions of child weight status and the subsequent weight gain of their children: A population-based longitudinal study. International Journal of Obesity, 41, 801-806.

Puhl, R. M., \& Brownell, K. D. (2001). Bias, discrimination, and obesity. Obesity Research, 9(2), 788-805.

Queally, M., Doherty, E., Matvienko-Sikar, K., Toomey, E., Cullinan, J., Harrington, J. M., ... On Behalf of the Choosing Healthy Eating for Infant Health (CHErIsH) Study Team. (2018). Do mothers accurately identify their child's overweight/obesity status during early childhood? Evidence from a nationally representative cohort study. International Journal of Behavioral Nutrition and Physical Activity, 15, 56-65.

Rankin, J., Matthews, L., Cobley, S., Han, A., Sanders, R., Wiltshire, H. D., \& Baker, J. S. (2016). Psychological consequences of childhood obesity: Psychiatric comorbidity and prevention. Adolescent Health, Medicine and Therapeutics, 7, 125-146.
Reilly, J. J., \& Kelly, J. (2011). Long-term impact of overweight and obesity in childhood and adolescence on morbidity and premature mortality in adulthood: Systematic review. International Journal of Obesity, 35(7), 891-898.

Remmers, T., van Grieken, A., Renders, C. M., Hirasing, R. A., Broeren, S. M. L., \& Raat, H. (2014). Correlates of parental misperception of their child's weight status: the 'Be Active, Eat Right' study. PLoS One, 9(2), e88931.

Rietmeijer-Mentink, M., Paulis, W. D., van Middelkoop, M., Bindels, P. J., \& van der Wouden, J. C. (2013). Difference between parental perception and actual weigh status of children: A systematic review. Maternal \& Child Nutrition, 9 (1), 3-22.

Rivera-Soto, W. T., \& Rodríguez-Figueroa, L. (2012). Childhood obesity among Puerto Rican children: Discrepancies between child's and parent's perception of weight status. International Journal of Environmental Research and Public Health, 9(4), 1427-1437.

Robinson, E. (2017). Overweight but unseen: A review of the underestimation of weight status and a visual normalization theory. Obesity Reviews, 18(10), 1200-1209.

Robinson, E., \& Sutin, A. R. (2017). Parents' perceptions of their children as overweight and children's weight concerns and weight gain. Psychological Science, 28(3), 320-329.

Rodrigues, D., Padez, C., \& Machado-Rodrigues, A. M. (2018). Prevalence of abdominal obesity and excess weight among Portuguese children and why abdominal obesity should be included in clinical practice. Acta Médica Portuguesa, 31(3), 159-164.

Sattler, K. M., Deane, F. P., Tapsell, L., \& Kelly, P. J. (2018). Gender differences in the relationship of weight-based stigmatization with motivation to exercise and physical activity in overweight individuals. Health Psychology Open, 5(1), 1-11.

Suls, J., Martin, R., \& Wheeler, L. (2002). Social comparison: Why, with whom, and with what effect? Current Directions in Psychological Science, 11(5), 159-163.

Szamreta, E. A., Qin, B., Ohman-Strickland, P. A., Devine, K. A., Stapleton, J. L., Ferrante, J. M., \& Bandera, E. V. (2017). Associations of anthropometric, behavioral, and social factors on level of body esteem in peripubertal girls. Journal of Developmental and Behavioral Pediatrics, 38, 58-64.

Tompkins, C. L., Seablom, M., \& Brock, D. W. (2015). Parental perception of child's body weight: A systematic review. Journal of Child and Family Studies, 24(5), 1384-1391.

Tschamler, J. M., Conn, K. M., Cook, S. R., \& Halterman, J. S. (2010). Underestimation of children's weight status: Views of parents in an urban community. La Clinica Pediatrica, 49(5), 470-476.

Turner, K. M., Salisbury, C., \& Shield, J. P. (2012). Parents' views and experiences of childhood obesity management in primary care: A qualitative study. Family Practice, 29(4), 476-481.

Twarog, J. P., Politis, M. D., Woods, E. L., Daniel, L. M., \& Sonneville, K. R. (2016). Is obesity becoming the new normal? Age, gender and racial/ethnic differences in parental misperception of obesity as being 'About the Right Weight'. International Journal of Obesity, 40, 1051-1055.

Warkentin, S., Mais, L. A., Latorre, M. R. D. O., Carnell, S., \& Taddei, J. A. A. C. (2018). Factors associated with parental 
underestimation of child's weight status. The Journal of Pediatrics, 94(2), 162-169.

Wijnhoven, T. M. A., van Raaij, J. M. A., Sjöberg, A., Eldin, N., Yngve, A., Kunešová, M., ... Breda, J. (2014). WHO European Childhood Obesity Surveillance Initiative: School nutrition environment and body mass index in primary school. International Journal of Environmental Research and Public Health, 11 (11), 11261-11258.

World Health Organization (WHO) (2000). Obesity: preventing and managing the global epidemic. In WHO (Ed.), Technical Report Series No 894. Geneva: WHO.

Yao, N. L., \& Hillemeier, M. M. (2012). Weight status in Chinese children: Maternal perceptions and child self-assessments. World Journal of Pediatrics, 8(2), 129-135.
Zhang, T., Cai, L., Jing, J., Ma, L., \& Chen, Y. (2018). Parental perception of child weight and its association with weight-related parenting behaviours and child behaviours: A Chinese national study. Public Health Nutrition, 21(9), 1671-1680.

How to cite this article: Rodrigues D, MachadoRodrigues AM, Padez C. Parental misperception of their child's weight status and how weight underestimation is associated with childhood obesity. Am J Hum Biol. 2020;e23393. https://doi. org/10.1002/ajhb.23393 